fSRM: A user-friendly R package for family data | 1

\title{
Getting the most out of family data with the R package fSRM
}

\author{
Lara $\operatorname{Stas}^{1}$ \\ Felix Schönbrodt ${ }^{2}$ \\ Tom Loeys ${ }^{1}$ \\ ${ }^{1}$ Ghent University \\ ${ }^{2}$ Ludwig-Maximilians-Universität München
}

\section{CITATION}

Stas, L., Schönbrodt, F., \& Loeys, T. (2015, March 2). Getting the Most Out of Family Data With the R Package fSRM. Journal of Family Psychology, 29(2), 263-275. http://dx.doi.org/10.1037/fam0000058 
fSRM: A user-friendly R package for family data $\mid 2$

\begin{abstract}
Family research aims to explore family processes, but is often limited to the examination of unidirectional processes. As the behavior of one person has consequences that go beyond that one individual, family functioning should be investigated in its full complexity. The Social Relations Model (SRM; Kenny \& La Voie, 1984) is a conceptual and analytical model which can disentangle family data from a round-robin design at three different levels: the individual level (actor and partner effects), the dyadic level (relationship effects) and the family level (family effect). Its statistical complexity may however be a hurdle for family researchers. The userfriendly R package $f S R M$ performs almost automatically those rather complex SRM analyses and introduces new possibilities for assessing differences between SRM-means and between SRMvariances, both within and between groups of families. Using family data on negative processes, different type of research questions are formulated and corresponding analyses with $f S R M$ are presented.
\end{abstract}

Keywords: Social Relations Model, Family Research, Structural Equation Model, R software. 
fSRM: A user-friendly R package for family data $\mid 3$

Family research aims to explore family processes, but is often limited to the examination of unidirectional processes. Taking the examination of how parents influence and shape their children (i.e., parenting) as an example, the historically dominating view has considered children to be passive receivers of these parental influences (Maccoby, 2003). It is however possible that children influence their parents too (Kerr \& Statin, 2003). The latter effects are called child effects. Both unidirectional views alone (i.e., parenting and child effects) are considered unable to capture the full complexity of family processes though. A bidirectional view is therefore gaining attention in the scientific literature (Cappa, Begle, Conger, Dumas, \& Conger, 2010; Cook, 2001; Majdandžić, Vente, Feinberg, Aktar, \& Bögels, 2012; Padilla-Walker, Carlo, Christensen, \& Yorgason, 2012). Bidirectionality emphasizes dynamics from the parent toward the child as well as from the child toward the parent (Kuczynski, 2003).

Despite the growing interest in bidirectional processes, popular methodologies are not always capable to capture these processes adequately and simultaneously (Cook, 2012). Since the behavior of one person within a family has consequences that go beyond that one individual (Cook, 2005) it is recommended to investigate the family functioning in its full complexity. The parent-child relationship for example is a specific dyad within a family as a whole and should not be viewed in isolation (Eichelsheim, Deković, Buist, \& Cook, 2009).

The Social Relations Model with roles (SRM; Kenny \& La Voie, 1984) is a conceptual and analytical model that allows family researchers to simultaneously investigate such complex processes (Kashy \& Kenny, 1990) while accounting for the different roles within a family. For example, the SRM assumes that when a mother rates her child on a given characteristic, the obtained score is not solely an objective description of this child's feature. Rather, it contains information about the mother who evaluates (i.e., the actor of the dyad), about the child being rated (i.e., the partner of the dyad), something about their unique relationship and information 
fSRM: A user-friendly R package for family data $\mid 4$

about their family culture. The SRM thus enables researchers to disentangle processes within family relationships at three different levels: the individual level (actor and partner effect), the dyadic level (relationship effect) and the family level (family effect) ${ }^{1}$. We refer the interested reader to Cook (2005); Kenny, Kashy, \& Cook (2006); Back \& Kenny (2010) for further details about the different SRM components.

The popularity of the SRM has risen over the last decade and has already been used to measure a wide range of variables like relationship satisfaction (Branje, Finkenauer, \& Meeus, 2007), attachment (Buist, Dekovic, Meeus, \& Aken, 2004), perceived support (Branje et al., 2002), trust (Delsing et al., 2003) and warmth or hostility (Manders et al., 2007) in families. The vast majority of these studies has focused on isolating and investigating the different sources of variability in family processes (Cook, 2005). Indeed, the SRM allows researchers to calculate how much variance in the observed measurements can be explained by every SRM component. These proportions serve as an indication of the relative importance of each component separately. For example, Eichelsheim et al. (2009) found that, averaged over studies, the actor effects generally explain the largest proportion of variance in the influence domain. Finding substantial actor variance for mothers, implies that some mothers experience more influence towards all family members than other mothers in other families. Besides the examination of the SRMvariances, one may gain important information from the SRM-means too. This has only been examined in a limited number of studies (e.g., Eichelsheim, Buist, Deković, Cook, Manders, \& Branje, 2011; Rasbash, Jenkins, O'Connor, Tackett, \& Reiss, 2011). Unfortunately, the computational details on the calculations of these SRM-means have not been described in current literature.

\footnotetext{
${ }^{1}$ Please note that the presented SRM and corresponding analyses are applicable to all sorts of groups with distuingishable roles.
} 
fSRM: A user-friendly R package for family data $\mid 5$

The rather complex analyses associated with the SRM and the lack of a user-friendly software package to specifically perform SRM analyses may have hindered family researchers to get the most out of their data. The current paper aims to bridge that gap and provides the description of an open-source package for the free R Environment for Statistical Computing ( $R$ Development Core Team, 2008). It should make those analyses much easier to perform. The package is called fSRM (Schönbrodt, Stas, \& Loeys, 2014), which stands for "family SRM". With this package, SRM analyses can be performed for groups defined by roles (e.g., families). For SRM-analyses with groups without roles (e.g., friends) the TripleR package was recently developed (Schönbrodt, Back, \& Schmukle, 2012; Schönbrodt, Back, \& Schmukle, 2014), but the required analyses fundamentally differ. In particular, for groups without roles the SRM-analysis is based on an ANOVA, which cannot be used for the SRM-analysis with roles. The latter analysis is typically based on a confirmatory factor analysis (CFA) approach. Therefore, the presented package builds on lavaan (Rosseel, 2012), a popular R package developed for structural equation modeling. The $f S R M$ package is especially tailored to the SRM-analysis. Provided the data follow a specific structure - which will be described later - one simple line of R-code in the $f S R M$ package may suffice to perform the required SRM-analysis. Its associated output provides overview tables of all parameters of interest, such as SRM variances, variance decompositions and reciprocities. In addition, several supplementary options are built in, such as the calculation of SRM-means, tests for differences between roles in terms of both variances and means. One can also add or relax restrictions of the default SRM model implemented in fSRM. Finally, the package also allows researchers to perform all those analyses in multigroup studies.

The structure of this paper is as follows. First, data on negative interactions in families, mimicking a study by Eichelsheim et al. (2011), are introduced. The goal of this study was to 
fSRM: A user-friendly R package for family data |6

investigate how patterns of negativity manifest in families with an adolescent with externalizing problem. In this paper, we will first describe several research questions about such processes that can be addressed by means of the SRM. Because estimation of the relevant parameters is typically performed by means of a structural equation model (SEM), the SRM is next casted within the SEM-framework. Readers not familiar with SEM may not be able to grasp all the technical details herein and may want to skip that section, but those more familiar with SEM may find it useful to understand the default assumptions made in the fSRM package. Next, we show with $f S R M$ how a few lines of R-code performs all the analyses needed to easily address the aforementioned research questions. The R-syntax and -output are explained in detail in the appendices, and require only a minimal amount of experience with R. Readers who wish to see additional or more complex examples can consult the supplementary materials. In those materials, two analyses described in detail in the reference book on dyadic data analysis by Kenny et al. (2006) are replicated step-by-step with fSRM. We end this paper with some additional technical features of the $f S R M$ package that may require more advanced SEM- and/or R-knowledge.

\section{Data}

\section{Motivating Example on Negativity in Families}

Eichelsheim et al. (2011) collected data from four-person families in a round-robin design. In such design, which is required for the application of the SRM, data are obtained on each individual's behavior, perceptions or emotions in relation to each of the other family members. In the study of Eichelsheim et al. (2011), four members of the same family (mother, father, target adolescent and sibling) reported on the amount of negativity they experienced in relation to each other. In total, these authors studied 120 Dutch four-person families with a target adolescent scoring above the externalizing behavior clinical norm scores on either the Child 
fSRM: A user-friendly R package for family data $\mid 7$

Behavior Check List (N = 47; CBCL; Achenbach, 1991) or the Youth Self Report (N = 73; YSR; Achenbach, 1991) and 153 four-person families of which the adolescents scored below the subclinical norm scores of both questionnaires. These two groups are further referred to as the problematic and non-problematic families, respectively. Because of confidentiality reasons, not the original data but mimicked data are used here, so results will deviate from the original paper. In first instance we will focus on the problematic families alone and their data are referred to in the $f S R M$ package as the clinical dataset. The dataset containing both groups is referred to as the two.groups dataset in the $f S R M$ package. All analyses presented throughout this paper can be replicated by the reader with the code provided in the appendices.

The primary purpose of these illustrating data is to explain the wealth of information that can be retrieved from such data with the SRM and how all that information can be easily obtained using fSRM.

\section{Descriptive Statistics}

In our motivating example, 12 dyadic measurements are obtained from every four-person family. Table 1 presents the mean and standard deviation per observed dyadic measurement on negativity in problematic and non-problematic families, respectively. Significant mean differences between these two types of families are most pronounced when the target adolescent is the rater or the person being rated. Interestingly, the highest amount of negativity in the problematic families is reported between the two siblings, in both directions. Furthermore, we observe the highest variability in those two dyadic measures too (e.g. variance target - sibling $=$ .96 ; variance sibling - target $=.87$ ).

Table A.2 in Appendix A shows the observed variance-covariance matrix for the 12 measurements in the problematic families: on the diagonal one finds the variance of each of the 
fSRM: A user-friendly R package for family data $\mid 8$

Table 1

Means, Standard Deviations, and ANOVA of Observed Dyadic Measurements

\begin{tabular}{lllll}
\hline Relationship & Problematic families & $\begin{array}{l}\text { Nonproblematic } \\
\text { families }\end{array}$ & $\mathrm{F}(1,271)$ & $\eta^{2}$ \\
\hline M-F & $1.63(.72)$ & $1.41(.62)$ & $7.41^{* *}$ & .03 \\
F-M & $1.55(.67)$ & $1.55(.61)$ & .00 & .00 \\
M-T & $1.77(.64)$ & $1.23(.54)$ & $56.06 * * *$ & .17 \\
T-M & $1.92(.78)$ & $1.48(.61)$ & $26.98^{* * *}$ & .09 \\
M-S & $1.71(.75)$ & $1.41(.68)$ & $11.73^{* * *}$ & .04 \\
S-M & $1.64(.83)$ & $1.63(.78)$ & .019 & .00 \\
F-T & $1.78(.69)$ & $1.40(.48)$ & $28.78^{* * *}$ & .10 \\
T-F & $1.87(.78)$ & $1.36(.48)$ & $43.71^{* * *}$ & .14 \\
F-S & $1.64(.60)$ & $1.59(.60)$ & .50 & .00 \\
S-F & $1.64(.79)$ & $1.63(.68)$ & .01 & .00 \\
T-S & $2.54(.96)$ & $2.14(.68)$ & $16.09 * * *$ & .06 \\
S-T & $2.56(.87)$ & $2.28(.86)$ & $7.02^{* *}$ & .03 \\
\hline
\end{tabular}

Note. T represents the target adolescent, $\mathrm{M}$ the mother, $\mathrm{F}$ the father and $\mathrm{S}$ the sibling. E.g., M-F $=$ mother rating father

12 measurements, while off the diagonal the covariances are shown. As correlations always lie between -1 and 1 , those are easier to interpret than covariances. Therefore the correlation matrix of the problematic families is shown in Table 2. The highest correlations are observed for dyadic measurements reported by the same two dyad members in relation to each other. Further, modest

Table 2

Correlation Matrix of Observed Dyadic Measurements of the ProblematicFamilies

\begin{tabular}{lllllllllllll}
\hline & M-F & F-M & M-T & T-M & M-S & S-M & F-T & T-F & F-S & S-F & T-S & S-T \\
M-F & & & & & & & & & & & & \\
F-M & .45 & & & & & & & & & & & \\
M-T & .07 & .02 & & & & & & & & & & \\
T-M & .19 & .15 & .35 & & & & & & & & & \\
M-S & .12 & .04 & .15 & .23 & & & & & & & & \\
S-M & .15 & .12 & .10 & .14 & .27 & & & & & & & \\
F-T & .12 & .10 & .28 & .18 & .05 & .00 & & & & & & \\
T-F & .26 & .12 & .23 & .31 & .12 & .13 & .53 & & & & & \\
F-S & .10 & .05 & -.05 & -.07 & .11 & .10 & .14 & .00 & & & & \\
S-F & .23 & .17 & -.04 & .02 & .15 & .27 & .14 & .14 & .37 & & & \\
T-S & .12 & .09 & .11 & .20 & .25 & .10 & .12 & .18 & .02 & .08 & & \\
S-T & .07 & .09 & .16 & .08 & .13 & .18 & .17 & .07 & .02 & .17 & .38 & \\
\hline
\end{tabular}

Note. T represents the target adolescent, $\mathrm{M}$ the mother, $\mathrm{F}$ the father and $\mathrm{S}$ the sibling. E.g., $\mathrm{M}-\mathrm{F}$ $=$ score of mother rating the father 
fSRM: A user-friendly R package for family data $\mid 9$

correlations are observed for dyadic measurements whose rater or person being rated are the same. All R-code to obtain the descriptive statistics above are provided in Appendix A.

\section{Translating Research Questions into the SRM-Framework}

Based on the observations from Tables 1 and 2, family researchers may be interested in a number of relevant questions that can be addressed with the SRM. Below is a non-exhaustive list of example questions that will be discussed throughout this paper. The first five questions pertain to the problematic families alone, while the last two questions consider comparisons between the problematic and non-problematic families:

Q1. The observed dyadic measurements (cfr. Table 1) can be disentangled into different SRM-components. But what is driving for example the variation in the dyadic measurements associated with adolescents with externalizing problem behavior? The highest variability is observed for the target-sibling relationship (see Table 1). Is this variation mainly due to differences in the way that target adolescents experience negativity in relation to all other family members (i.e., actor variance of the targets)? Some target adolescents may experience a lot of negativity in relation to all other family members, whereas others do not experience much negativity in relation to all other family members. Or is it due the differences in the way that negativity in relation with the siblings is experienced (i.e., a partner variance of the siblings)? Is it due to the unique relationship between these target adolescents and siblings (i.e., a relationship variance)? Or do families differ in general in the amount of negativity (i.e., a family variance)? Therapists may want to identify the importance of each of these SRM components since they may want to intervene in those processes that vary between families, and might thus be subject to change. 
fSRM: A user-friendly R package for family data | 10

Q2. Is the amount of negativity that the target adolescent is experiencing with other family members (i.e., actor effect target adolescent) associated with the amount of negativity that the other family members experience in relation to him/her (i.e., partner effect target adolescent)? Similar questions can be raised for the other roles in the family. These associations are sometimes referred to as generalized or individual reciprocities. We indeed noted modest correlations for dyadic measurements whose rater or person being rated are the same, but the individual reciprocities themselves cannot be directly read from Table 2 since the observed measurements are composed by different SRM-components.

Q3. Is the unique amount of negativity that the target child is experiencing in relation to the sibling also associated with the unique amount of negativity that the sibling is experiencing from him/her, independent of the more general characteristics of both children and the family? Such associations are referred to as dyadic reciprocities. Note that these cannot be read directly from the correlation matrix presented in Table 2 either because those correlations do not have the actor and partner effects filtered out yet.

Q4. Are parents (respectively children), similar in the way they experience negativity in relation with all other family members (i.e., their actor effects), and in the way they are experienced by other members (i.e., their partner effects)? In other words, do we observe similarities within the same generation (i.e. intragenerational similarities)?

Q5. Why is more negativity reported when an adolescent with externalizing problem behavior is involved (cfr. Table 1)? Are the interactions with this target adolescent more negative because he/she typically identifies all his/her family members as negative (i.e., actor effect target adolescents)? As an alternative, do all other family members experience a lot of negativity in relation to this target (i.e., partner effect target adolescent)? Or is it due to a unique 
effect of a particular family relation (i.e., relationship effect)? While Q1 focusses on the variability of the SRM components, Q2 to Q4 on correlations of SRM-components, Q5 is concerned with the mean of the SRM components.

Q6. Are the sources of variation in negativity the same in problematic and nonproblematic families? Do target adolescents in problematic families, for example, show larger variation in the amount of negativity they experience in relation to all their family members than their matching adolescents in the non-problematic group (i.e., different actor variance of the targets between both groups)?

Q7. Which SRM components account for the differences in mean negative processes between the problematic and nonproblematic families in Table 1? Is the overall amount of negativity experienced in problematic families equal to the amount experienced in nonproblematic families (i.e., mean difference in family effects between these two groups). Is the amount of negativity that target adolescents in problematic families experience in relation to all their family members the same as the amount experienced by the matching adolescents in the non-problematic group (i.e., mean difference in actor effects)?

\section{The SRM within the SEM-Framework}

As notedin the introduction, one of the strengths of the SRM is that it allows to disentangle the dyadic measurements at three different levels: the individual, the dyadic and the family level. In this section, we elaborate on the standard assumptions of the SRM and discuss how each of the questions raised in the previous section can be formulated in terms of the SRM parameters.

Let $\mathrm{X}_{\mathrm{ij}}$ represent the score of the person with role $i(i=$ mother, father, target, sibling) rating person with role $j(j=$ mother, father, target, sibling). The SRM then assumes that each 
dyadic measurement $X_{\mathrm{ij}}$ is a function of four latent effects: a family effect, an actor effect, a partner effect and a relation-specific effect. Figure 1 shows the four-person model with these SRM components specified as latent variables. The subscripts $\mathrm{m}, \mathrm{f}, \mathrm{t}$ and $\mathrm{s}$ refer to the mother, father, target, and sibling, respectively. The dyadic measure of the target adolescent rating the sibling (i.e., $\mathrm{X}_{\mathrm{ts}}$ ), for instance, is predicted by the family-effect, the actor effect of the target, the partner effect of the sibling, and the target-sibling relation-specific effect. Therefore, arrows point from those four latent variables towards this dyadic measurement. Typically all paths from the SRM components to the observed scores are fixed to one in the CFA-approach (for more details see Kenny et al., 2006, p. 236). The fSRM package makes that assumption too. The variances of the SRM-components on the other hand are estimated and therefore an asterisk is displayed in Figure 1. For a clear description of the different matrices involved in the CFAformulation, we refer the interested reader to Kenny et al. (2006).

Alternatively one can view the SRM as a multilevel model (Rasbash et al., 2011; Snijders \& Kenny, 1999). The score $\mathrm{X}_{\text {tsk }}$ of the target adolescent rating its sibling in family $k(k=1, \ldots, \mathrm{N})$ is the sum of a family effect $\mathrm{f}_{\mathrm{k}}$, an actor effect $\mathrm{a}_{\mathrm{tk}}$, a partner effect $\mathrm{p}_{\mathrm{sk}}$, a relationship effect $\mathrm{r}_{\mathrm{tsk}}$ and a residual error term $\varepsilon_{t s k}$ :

$$
X_{t s k}=f_{k}+a_{t k}+p_{s k}+r_{t s k}+\varepsilon_{t s k}
$$

with $\quad f_{k} \sim \mathrm{N}\left(\mu_{\mathrm{fam}}, \sigma_{\mathrm{fam}}^{2}\right), \quad a_{t k} \sim \mathrm{N}\left(\alpha_{t}, \sigma^{2}\right.$ act, $\left.\mathrm{t}\right), \quad p_{s k} \sim \mathrm{N}\left(\beta_{\mathrm{s}}, \sigma_{\mathrm{par}, \mathrm{s}}^{2}\right), \quad r_{t s k} \sim \mathrm{N}\left(\gamma_{\mathrm{ts}}, \sigma^{2}\right.$ rel, ts $) \quad$ and $\varepsilon_{t s k} \sim \mathrm{N}\left(0, \sigma^{2}\right.$ res $)$. Note that similar equations can be formulated for each of the 11 other dyadic measurements. The parameters $\sigma_{\text {fam }}^{2}, \sigma_{\text {act,t }}^{2}, \sigma_{\text {par,s }}^{2}$ and $\sigma_{\text {rel,ts }}^{2}$ represent the family variance, actor variances of target adolescents, partner variances of siblings and their relation-specific variances, respectively. Once we have estimated each of these variances from the model, we will be able to answer question Q1 on what is driving the variation in that outcome. Note that in the fSRM 
package, the model in Equation (1) is fitted within a CFA framework since it allows greater flexibility in constraining and updating the model than the multilevel framework.

It should be noted that with only one indicator/measurement per construct it is impossible to separate the relationship effect from the residual (Cook, 1994). Separation of those effects requires the use of at least two indicators/observations for each dyadic measurement. With two indicators instead of one, the observed variance-covariance is a $24 \times 24$ matrix instead of a $12 \times 12$ matrix. Given the relatively small sample sizes typically encountered in family research, the use of two indicators can however result in a low subject-per-variable ratio. As the latter may lead to unstable estimates of the SRM components (Cook, 1993, 1994), the use of two indicators is often avoided. Eichelsheim et al. (2012) measured negativity using the negative interaction subscale of the Network of Relationship Inventory (NRI; Furman \& Buhrmester, 1985), consisting of 6 items that reflect hostile behavior as well as (the frequency of) conflicts to be answered on a 5point scale. In the case of one indicator, one does simply take the average of those 6 items. For the case of 2 indicators one could take for example the average of the first 3 items as the first indicator, and the average of the last 3 items as the second indicator. $f S R M$ allows both for one or multiple indicators. If only a single indicator is specified for each dyadic measurement, the relation-specific variance presented by the $f S R M$ package will also contain the residual error variance. With two indicators, the residual error will automatically be separated from the relationspecific effect. In the four-person model with a single indicator, the SRM-analysis results in 21 SRM variances: one family variance, four actor variances, four partner variances and twelve relationship variances. As will be illustrated in the next section, the package will by default provide estimates of all these SRM-variances together with a decomposition of every dyadic measure showing the relative contribution of each component. When two indicators are provided, the $f S R M$ package additionally assumes 24 residual variances (i.e., one residual variance for each 
fSRM: A user-friendly R package for family data | 14

of the $12 \times 2$ dyadic measurements). In this paper we will focus on settings with a single indictor, but additional examples for two indicators can be found in the supplementary material.

In addition to the SRM-variances, reciprocities can be specified as well. These reciprocities are shown in Figure 1 as double-headed arrows. Two types of reciprocities are assumed by default in the $f S R M$ package: reciprocities at the individual level (needed to address Q2) and reciprocities at the dyadic level (needed to address Q3). At the individual level, generalized reciprocities are characterized as the correlation between a person's actor and partner effect. At the dyadic level, reciprocities are marked as the correlation between the relationship effects of the two members of the same dyad. In the four-person model, there are four generalized and six dyadic reciprocities. The SRM optionally allows researchers to request intragenerational similarities (needed to address Q4) between family members (Cook, 2000; De Mol et al., 2010; Kashy \& Kenny, 1990), but those are not assumed by default. Intragenerational reciprocities are characterized by the correlations of the actor (partner) effects of family members from the same generation. In sum, applying the defaults of the fSRM package in the four-person model with a single indicator, the SRM assumes 31 unknown parameters (21 variances and 10 reciprocities) while $12 \times 13 / 2=78$ (co-)variances are observed. In the CFA-approach implemented here, this model is then overidentified with 47 degrees of freedom (df). The goodness-of-fit of the model can be assessed by comparing the observed variance-covariance matrix (as shown for example in Appendix A Table A.2) with the model-implied variance-covariance matrix. More details on model fit assessment are provided in Appendix C. When two indicators are specified for each dyadic measurement, $f S R M$ will automatically correlate errors within the same indicator to capture measurement method variance due to the use of the same items (as suggested by Kenny, Kashy \& Cook, 2006, p.247). In that case, the default four-person model in the fSRM package has 
fSRM: A user-friendly R package for family data $\mid 15$

187 unknown parameters (21 SRM-variances, 10 reciprocities, 24 residual variances and 132 method covariances) while $24 \times 25 / 2=300$ (co-)variances are observed (i.e., $\mathrm{df}=113$ ).

So far, the SRM has been discussed in terms of (co-)variances, but each of these variances measure deviations around a mean effect. Equation (1) makes this more explicit. The parameters $\mu_{\text {fam }}, \alpha_{t}, \beta_{\mathrm{s}}$ and $\gamma_{\mathrm{ts}}$ describe an average family effect, average actor effect for the target adolescents, average partner effect of the siblings, and their average relationship effects, respectively. In the four-person model with one indicator per relationship, we have in total 21 SRM-means (1 family mean, 4 actor means, 4 partner means and 12 relation-specific means), but only 12 means are observed (see Table 1). Therefore, restrictions are commonly applied such that the mean actor effects sum to zero, the mean partner effects sum to zero, and the mean SRM relationship effects sum to zero for a given actor or a given partner. Both actor and partner means then represent deviations from the overall grand-mean (i.e., the family mean; Kenny et al., 2006; Eichelsheim et al., 2009; Rasbash et al., 2011). In the fSRM package, this particular parameterization is used too, and the SRM-means are estimated (cfr. Q5) by adding a mean structure to the SRM basic covariance structure. Technically, the above described restrictions on the SRM-means are implemented by adding equality constraints. When these SRM-means are requested by the user, $f S R M$ actually performs a CFA with structured means. Note that when two or more indicators are used for each relationship measure, the $f S R M$ package assumes common means for each SRM component since the indicators are typically replications of the same underlying construct (e.g., two indicators are assumed to measure the same family mean). This assumption can easily be checked by comparing the fit of the model with and without means specified. The latter approach indeed corresponds to assuming different means for each indicator. The difference between the two chi-squares of those models is itself a chi-square with $12 \mathrm{df}$ (in case of a four-person model with two indicators). 
fSRM: A user-friendly R package for family data $\mid 16$

When multiple groups are involved (cfr. Q6 and Q7) the default SRM is basically fitted to the data of each group separately.

\section{SRM-Analyses with the $f S R M$ Package}

This section illustrates the use of the $f S R M$ package by means of the aforementioned data on negative processes within families and the seven research questions raised. First, the basics on installing and loading R-packages, and on importing data in R are briefly described. Next, it is demonstrated how to perform SRM analyses with $F S R M$ along with a discussion of the associated output.

\section{Installing and Loading the Package}

The $f S R M$ package was built under $\mathrm{R}$ version 3.1. Hence, this version or more recent versions of $\mathrm{R}$ are required to use fSRM. In order to install and load $f S R M$, write install.packages ("fSRM") and library ("fSRM") on the command line in R, respectively.

\section{The Pop-Up Window: Easily Inserting and Transforming a Dataset}

SRM data are typically organized in a wide format such as in the left panel of Figure 2 (i.e., each column representing one observed dyadic measurement, and one row for each family). However, in order to use $f S R M$, the data need to be organized in a long format, such as in the right panel of Figure 2. The long data format consists of at least four columns: a column representing the role of the actor, a column for the role of the partner, one with the observed score and a column representing the family identification number. If multiple indicators and/or groups are used, additional columns defining the indicator and/or group are needed. The fSRM package can transform a dataset from a wide format into such long format for the user, provided 
that the variable names of the original dataset follow some restrictions. In the left panel of Figure 2, the variable names are constituted by different characters, with the first character indicating the role of the actor, the second character the role of the partner and the last three the indicator of the construct being measured (i.e., "neg" represents the construct negative interactions).

In order to be able to use this transformation tool, the number of characters used for the identification of the rater and the person being rated should be the same. For instance, the negativity rating of the mother ("M") about the target adolescent ("T") can be labeled "MTneg", but not "MOTneg". The characters for the roles in the variable names will be used later in the output as labels for the different roles. Note that, when multiple indicators are present, the number of characters specifying these indicators in the variable name, should be the same. For example, if the first indicator of a dyadic rating of the mother about the target adolescent is called "MTneg1" or "MTxxx", the second indicator should have variable name "MTneg2" or "MTzzz", respectively.

To transform the original wide format dataset, complying with those variable name restrictions, into a dataset with long format, one should next type the command import () on the R command line. A pop-up window then appears (see Figure 3), which allows the user to identify the location of the original dataset (which should have extensions .txt, .csv or .sav). After importing the datafile, the label of the button will change into "datafile added".

Next, one should click the button "Transform my data". A new window will appear (see Figure 4). First, the column number of both the family and the group identification variable in the original dataset should be provided, if present. In the absence of a family identification variable in the original dataset, $f S R M$ automatically creates the required family identification variable. Next, the user needs to specify the columns in the original dataset which contain the dyadic 
measurements for the SRM analyses ${ }^{2}$. The enumerated columns need to be separated with a comma, consecutive columns are defined by means of colon (e.g., 1:12 defines the first until the twelfth column in the original clinical.wide dataset ${ }^{3}$, containing the 12 dyadic measurements). Finally, the user is asked to define the position of the characters of the rater in the previously selected measurements, the person being rated and the indicator (if present), which should be filled in for the clinical dataset as "1 to 1 ", "2 to 2 " and empty, respectively. By clicking on "OK" the user returns to the main window.

In this main window the user can agree with the default labels of the SRM components (i.e. "FE" for family effect, " $A$ " for actor effect, " $P$ " for the partner effect and " $R$ " for the relationship effect) by clicking on "Confirm output format" or he/she can specify alternative labels. The "Confirm output format" button than changes into output "Outputformat confirmed". By clicking on "OK" at the bottom of the window the transformed dataset will be saved as a hidden variable. In this dataset the variables defining the actors, partners, groups and indicators are called actor.id, partner.id, group, ind and family.id, respectively. In order to access this hidden variable, the user needs to call the function getImport (). For example, after entering mydata <- getImport () on the $\mathrm{R}$ command line, the transformed dataset can be used as mydata in the $\mathrm{ESRM}($ ) function.

\section{The Standard Four-Person Model with one Indicator and one Group}

Using the clinical dataset in long format from the fSRM package, this section first illustrates how the SRM (co-)variances can easily be obtained using the main function in the package the $\operatorname{ESRM}()$ function. In this function, the so called "formula syntax" together with the

\footnotetext{
${ }^{2}$ When multiple constructs are of interest, a separate input dataset should be created for each construct.

${ }^{3}$ The content of this dataset in wide format is identical to the clinical dataset, which is in long format. The clinical.wide dataset is available in the online supplemental material, as well as within the fSRM package.
} 
name of the dataset needs to be specified. In particular, the formula is constituted as DV actor.id * partner.id |family.id, where DV is the name of the variable that contains the value of the construct of interest, actor.id is the name of the variable which specifies the role of the actor, partner.id is the name of the variable which specifies the role of the partner, and family.id is the name of the variable that identifies the family (see also the right panel of Figure 2, where DV is the "value" column here). So here, one specifies:

fSRM.4.1 <- fSRM(neg actor.id*partner.id | family.id, data = clinical)

This single line of R-code and associated output are presented in Appendix B in the section "Code 1: Basic SRM analyses". In the output the heading "SRM with roles" shows the label names of the roles of the different family members together with the dependent variable. Next, a brief model summary is provided under the heading "Model Summary" (with the earlier described $47 \mathrm{df}$ ), followed by the fit indices under the header "Model Fit", revealing an excellent fit here ${ }^{4}$. Under the header "variance decomposition" the estimates of the SRM variances are presented. In the first column the different labels of the SRM components are shown. The character(s) preceding the dot represent the labels of the different SRM components as confirmed in the pop-up window. The characters following the dot define the role of the family member (e.g., A.m. denotes the actor effect of the mother, P.f. the partner effect of the father). In case of relationship effects the character after the first dot represents the rater in the dyadic measurement and the character after the second dot the person who is being rated (e.g., the relationship effect of the mother towards the father is R.m.f). The second column with heading "estimate" shows the variance estimates of each SRM component. The remaining columns show the standard errors (i.e., the se-column), the z-value (i.e., the z-column), the 1-sided p-value

\footnotetext{
${ }^{4}$ For guidance on the model fit assessment the interested reader is refered to Appendix C.
} 
fSRM: A user-friendly R package for family data | 20

(i.e., the p.value-column), the significance level (with the usual convention for 1 to 3 asterisks denoting $p<0.05, p<0.01$, and $p<0.001$, respectively) and the 2 -sided $90 \%$ confidence interval conform with the one-sided p-values (i.e., the ci.lower-and ci.upper-column).

By default, $f S R M$ will report one-sided p-values and two-sided $90 \%$ confidence intervals for the variance components ${ }^{5}$.

To answer research question Q1 (i.e., "What is driving the variation in the dyadic measurements associated with the adolescents with externalizing problem behavior?"), one finds significant variation across problematic families in the amount of negative interaction the target adolescent reports with all his/her family members (i.e., actor variance target child $=0.080, p<$ .05). Likewise, across these families a significant difference exists in the amount of negative interactions all his/her relatives report in relation to him/her (i.e., partner variance target child $=$ 0.072, $p<.05)$. Furthermore, all the SRM relationship effects are significant $(p<.001)$.

In order to gain a better understanding of the size of each SRM component the section "Relative variance decomposition" is printed in the output. The columns reflect the relative importance of each SRM component both per observed relationship, as well as averaged over the different relationships. Note that summing up the different components results in $100 \%$. As there is only one indicator per relationship measure in this example, the residual variance is contained in the relationship-specific variance and therefore set to zero. A graphical representation of this table can be obtained by applying the plot function to the fitted SRM object (see appendix B: Code 1). From the resulting figure (illustrated by Figure 5), one can easily see that on average the majority of the variance is explained by the relationship effects, followed by both the partner and the actor effects. Likewise, this figure clearly reflects that the variation on the reported negativity

\footnotetext{
${ }^{5}$ The default confidence level is set for all parameters to $95 \%$, except for the SRM variance components. The latter are set to $90 \%$, conform the one-sided p-values for the variance components.
} 
fSRM: A user-friendly R package for family data | 21

between two siblings is mainly an effect of their unique relationship. Therefore, these data may indicate that in therapy it may be useful to focus on unique relationships rather than on characteristics of individual family members. One should realize however that with one indicator here, the residual error variance is part of the relationship variance too.

In the last two parts of the output, the generalized and dyadic reciprocities are displayed ${ }^{6}$. Here, the "estimate"-column contains the covariances, the "r"-column the correlations. The corresponding output can be used to answer Q2 and Q3, respectively. In this study no significant correlation. between the actor and partner effects is found for any role $(p>.05)$. For example, the amount of negativity that the target child is experiencing with all other family members (i.e., actor effect of the target adolescent) is not associated with the amount of negativity that the other family members experience with him/her (i.e., partner effect of the target adolescent). At the dyadic level on the other hand, significant reciprocity effects are observed for the relationships of both children with the father (T-F: $\mathrm{r}=.263, p<.05$ and S-F: $\mathrm{r}=.286, p<.05)$, between the parents $(\mathrm{r}=.300, p<.05)$. and between the siblings $(\mathrm{r}=.300, p<.05)$. The latter indicates that the more (less) negativity the target adolescent is experiencing with the sibling, the more (less) negativity the sibling will report in relation to this child, independent from their actor and partner effects.

Intragenerational similarities are not assumed by default and must be requested by simply inserting the IGSIM-argument to the ESRM() function. This option assumes correlation between the actor and partner effects of members of the same generation. One can perform an omnibus test to test the overall presence of intragenerational similarities by comparing the chi-square test of the default model to this model. Additionally each intragenerational similarity can be

\footnotetext{
${ }^{6}$ Please note that it is only useful to interpret these reciprocities when both corresponding variance component differ significantly from zero.
} 
fSRM: A user-friendly R package for family data | 22

separately assessed at the 5\% significance level with the standard Wald-test. The R-code and corresponding output can be found in Code 2 of Appendix B. In this dataset, no intragenerational similarities were withdrawn $(p>.05)$.

In order to obtain information about the mean levels of the SRM components, the argument means=TRUE needs to be added to the fSRM() function (see code 3 in Appendix B). The average negativity score across families equals 1.854 . The highest levels of negativity are related with characteristics of the target adolescent as he/she experiences the other family members as most negative (i.e., mean actor effect $\mathrm{T}=0.356, p<.001$ ). These adolescents are also experienced as most negative by their relatives (i.e., mean partner effect $\mathrm{T}=0.299, p<$ .001). Finally, the target-sibling and sibling-target relation-specific effects are among the highest too. Figure 6 helps understanding the decomposition of the dyadic measurements into these SRM components. This graphical representation of the mean SRM-components can be requested by adding the option means = TRUE to the plot function (see code 3 in Appendix B) and is shown in Figure 6. The 12 labels on the $\mathrm{X}$-axis represent the 12 dyadic measurements, with the character before the hyphen representing the rater (i.e. the actor of the dyad is represented by a solid line) and the character after the hyphen indicating the person being rated (i.e., the partner of the dyad is represented by a dashed line). For instance, we noted before that on average adolescents with externalizing problem behavior rated their mothers as negative (cfr. Table 1). The tenth set of arrows in Figure 4 disentangles this mean score into its exact etiology (i.e., the SRM components). The target adolescent typically reports a high average level of negativity in relation to everybody (i.e., actor effect target $=0.356, p<.001$ ), which can be seen at his solid line. Mothers on the other hand are in general rated as less negative (i.e., partner effect mother = 0.226, $p<.001$ ), as the dashed line lowers the score. And, although not significant, target 
fSRM: A user-friendly R package for family data | 23

adolescents experience less unique negativity in relation to their mother since the dotted line lowers the score even further (relationship target-mother $=-0.068, p=.095$ ). In sum, the elevated score is mainly due to the adolescents' general sense of negativity in relation to all family members, instead of the specific mother-child relationship.

It can further be interesting to formally test whether the mean SRM components differ significantly by role. For example, is there a difference in the amount of negativity that each of the family members report towards their other family members (i.e., comparing actor effects)? Typically this is done by comparing the chi-squares of a constrained model (which assumes all actor means to be zero, for example) with an unconstrained model (Kenny et al., 2006; Eichelsheim et al., 2011; Rasbash et al., 2011), but such approach is computationally very slow as it requires fitting a constrained model for each type of SRM component. Therefore, alternative methods were explored to test the equality of mean effects effectively and accurately ${ }^{7}$. Since this Wald-test is computationally much faster (it only requires fitting the unconstrained model), this approach is included in $f S R M$. Testing whether all the actor, partner or relationship means are equal across roles can easily be done by the function equalmeans (). Additionally, one can ask for pairwise comparisons of the means of the actor (and partner) effects between two roles. This is done by adding pairwise = TRUE in the ESRM() function. For an example and additional comments on the latter two functions, please consult code 4 in appendix B.

\section{Examining and Comparing Multiple Groups}

The package can also be used to compare two groups (for example, the problematic versus the non-problematic families), using the group option. In analogy to single group analyses the

\footnotetext{
${ }^{7}$ Using simulations, a test based on the multivariate Wald-test was evaluated and compared with the results of the chi-square difference test (see supplementary material). Both approaches typically produce similar results.
} 
fSRM: A user-friendly R package for family data | 24

output presents a model summary and fit indices, followed by the variance decomposition, relative variance decomposition, generalized and dyadic reciprocities for each of the groups separately.

In such a multiple group setting, it is interesting to investigate whether the variances and/or the means of the SRM components differ between groups. Typically, such comparisons are based on a chi-square difference test (Eichelsheim et al., 2011). But such approach requires a large amount of constrained models (one for each comparison) to be compared with the unconstrained model that allows all means and variances to differ between groups. The Waldtest, with the standard error of the difference calculated following the delta-method, requires only one model to be fitted and is used in $f S R M^{8}$.

By simply adding the option diff = TRUE to the fSRM() formula the output presents the differences between groups in terms of SRM variances. The difference in SRM means are requested by additionally specifying the means option. The corresponding R-code and -output can be found in code 5 of Appendix B and can be used to answer Q6 and Q7.

The differences in the sources of variation between problematic and non-problematic families are situated at the dyadic level (i.e., significant differences in the variance of SRM relation-specific effects) but not at the individual or family level. In terms of the means, the adolescent with externalizing problems experiences more negativity in relation to all his/her family members than the matching adolescent in the nonproblematic group (i.e., $\mathrm{M}=0.262, p<$ .001). Likewise, a significant difference in family means is observed between problematic (group 1) and nonproblematic families (group 2), with problematic families overall reporting more than nonproblematic families $(\mathrm{M}=0.209, p<.001)$.

\footnotetext{
${ }^{8}$ Simulation studies again showed that the test based on the chi-square differences and on the Wald test using the delta method are comparable in terms of power and Type I error (cfr. supplementary material).
} 
fSRM: A user-friendly R package for family data $\mid 25$

\section{Additional Features of $f S R M$}

So far we presented the main functionalities of the default SRM in fSRM. The different features of the package enable the users to investigate their family data from a round robin design with a minimum of effort. When the default settings of the standard model yield a reasonable fit, the user can immediately focus on the interpretation of the results. The output from the $f S R M$ package provides the most relevant pieces from the CFA in an organized and clear fashion. When the standard SRM is not fitting well, one may try to find out the reasons by exploring the socalled modification indices, and assess whether the fit can be improved with some additional assumptions. To identify the origin of the bad fit, the function $\bmod ()$ in $f S R M$ can be used and will yield a listing of all possible adjustments with a modification index exceeding 10. Some familiarity with the notational conventions in lavaan (Rosseel, 2012) is needed though to understand this listing. Moreover, any adjustments to the model should not be solely based on statistical grounds, but have some theoretical rationale. The add-option in the $f S R M$-function can be used to add one or more adjustments to the standard model. It is possible to request the lavaan code behind the ESRM() function applied to a specific model by using cat (object\$syntax), where object is the name assigned to the fitted fSRM object. Users familiar with lavaan may start from that code to go beyond the features that are provided by fSRM. One could think about several extensions.

We focused above on the standard design with four people in all families and a single indicator. The supplementary material contains an example with two indicators and illustrates how the error variance can be separated from the relationship variance. All the described SRMparameters can simultaneously be estimated when there are more than four persons too. But in order for the SRM-parameters of a three-person model to be identified, some components of the 
fSRM: A user-friendly R package for family data $\mid 26$

model have to be dropped (Kenny et al., 2006, p. 245). As in most studies published so far the family variance tended to be small, the $f S R M$ package therefore drops by default the family variance in the three-person model. This allows all other co-variances to be estimated. However, the user can specify alternative models by adding the drop-argument to the fSRM() function. One can choose to alternatively drop all the actor effects (and corresponding reciprocities), all the partner effects (and corresponding reciprocities) or the generalized reciprocities by adding drop= with "actor", "partner" or "GR", respectively. We refer the interested reader to Kenny et al. (2006, p. 250) for a clear description when each of these options make sense. A detailed example of a three person analysis can be found in the online supplementary material too.

To avoid listwise deletion in the presence of missing data, the default in fSRM is set to Full Information Maximum Likelihood (FIML) rather than Maximum Likelihood. One may also choose not to use the maximum likelihood estimators for the SRM-parameters, but rely on alternative options in lavaan such as Generalized Least Squares estimators, or rely on bootstrap standard errors rather than the conventional standard errors by adding estimator = "GLS" or se = "boot" to the fSRM() function, respectively. Recently, it was discussed how to include covariates within the SRM in a Bayesian multilevel framework (Lüdtke et al., 2013), but such adjustments are also possible within the traditional SEM-framework.

Negative SRM-variances are not set to zero by default like other common software packages (e.g., EQS) as they might be an indicator for model misspecification. Nonetheless, this can be achieved by using the noNegVar option of the fSRM() function. The default uses noNegVar $=$ FALSE, and will provide the output from the original model with possible negative variances. Adding noNegVar $=$ TRUE to the ESRM() function will automatically constrain negative SRM-variances to be equal or greater than zero. 
Furthermore, an option is included that enables the user to test whether the social relations model fits the data better than a null model. In such a null model the (co-)variances of the different components are equal across roles, and the mean actor, partner and relationship effects equal to zero. The R-code for such model comparison can be found in code 6 of Appendix B. When a significant difference is found, one can conclude that the roles in the family really matter.

Finally note that like any other software package, fSRM may produce warning messages which may point to model misspecification amongst other. These are not inherent to the package but to the data and/or the fit with the specified model.

\section{Conclusion}

This paper provides a user-friendly package that covers the majority of the published SRM-analyses. Avoiding a multitude of model comparisons, it is computationally fast. In principal, every SEM-software can perform these SRM analyses. But while these software packages require a detailed knowledge of both the syntax and CFA, this is not the case with the fSRM package. Indeed, with $f S R M$, a single line of code may suffice to fit even complex Social Relations Models. Moreover the software is freely available. It could be useful if family researchers who publish SRM analyses in the future provide the complete $f S R M$-output as supplemental material to their paper.

Our hope is that family researchers who want to analyze their data from a round-robin design with the SRM in the future find it an easy tool that allows them to get the most out of their data and to communicate about their results. 
fSRM: A user-friendly R package for family data $\mid 28$

\section{Acknowledgments}

The authors would like to thank Yves Rosseel for his technical support with the use of lavaan in fSRM. We are also grateful to David Kenny and William Cook for stimulating discussions on the different features of fSRM and for giving permission to use the attachment data. Finally, we also like to thank Veroni Eichelsheim, Susan Branje and the whole RADAR-team for granting us access to their EQS scripts and their negativity data. Tom Loeys would like to thank the Research Foundation Flanders (FWO) for financial support (Grant G020115N).

\section{References}

Achenbach, T.M. (1991). Manual for the Child Behavior Checklist 4-18 and 1991 Profiles. Burlington, VT: University of Vermont, Department of Psychiatry.

Back, M. D., \& Kenny, D. A. (2010). The Social Relations Model: How to understand dyadic processes. Social and Personality Psychology Compass, 4, 855-870. doi:10.1111/j.17519004.2010.00303.x

Branje, S. J. T., Aken, M. A. G. van, \& Lieshout, C. F. M. van. (2002). Relational Support in Families with Adolescents. Journal of Family Psychology, 16(3), 351-362. doi:10.1037//0893-3200.16.3.351

Branje, S. J. T., Finkenauer, C., \& Meeus, W. H. J. (2007). Modeling Interdependence Using the Social Relations Model: The Investment Model in Family Relationships. In N. Card, J. Seli, \& T. D. Little (Eds.), Modeling interdependent data in developmental psychology. Mahwah, NJ: Card.

Buist, K. L., Dekovic, M., Meeus, W. H., \& Aken, M. A. G. van. (2004). Attachment in Adolescence:: A Social Relations Model Analysis. Journal of Adolescent Research, 19(6), 826-850. doi:10.1177/0743558403260109 
fSRM: A user-friendly R package for family data | 29

Cappa, K. A., Begle, A. M., Conger, J. C., Dumas, J. E., \& Conger, A. J. (2010). Bidirectional Relationships Between Parenting Stress and Child Coping Competence: Findings from the Pace Study. Journal of Child and Family Studies, 20(3), 334-342. doi:10.1007/s10826010-9397-0

Cook, W. L. (1993). Interdependence and the Interpersonal Sense of Control: An Analysis of Family Relationships. Journal of Personality and Social Psychology, 64(4), 587-601. doi:10.1037/0022-3514.64.4.587

Cook, W. L. (1994). A Structural Equation Model of Dyadic Relationships Within the Family System. Journal of Consulting and Clinical Psychology, 62(2), 500-509. doi:10.1037/0022-006X.62.3.500

Cook, W. L. (2000). Understanding Attachment Security in Family Context. Journal of Personality and Social Psychology, 78(2), 285-294. doi:10.1037//0022-3514.78.2.285

Cook, W. L. (2001). Interpersonal Influence in Family Systems: A Social Relations Model Analysis. Child development, 72(4), 1179-1197. doi:10.1111/1467-8624.00341

Cook, W. L. (2005). The SRM Approach to Family Assessment. European Journal of Psychological Assessment, 21(4), 216-225. doi:10.1027/1015-5759.21.4.216

Cook, W. L. (2012). Whole-family vs. SRM assessments. Unpublished manuscript. Vermont University.

De Mol, J., Buysse, A., \& Cook, W. L. (2010). A family assessment based on the social relations model. Journal of Family Therapy, 32(3), 259-279. doi:10.1111/j.14676427.2010.00504.x

Delsing, M. J. M. H., Oud, J. H. L., De Bruyn, E. E. J., \& Van Aken, M. A. G. (2003). Current and recollected perceptions of family relationships: The social relations model approach 
fSRM: A user-friendly R package for family data $\mid 30$

applied to members of three generations. Journal of family psychology, 17(4), 445-459. doi:10.1037/0893-3200.17.4.445

Eichelsheim, V. I., Buist, K. L., Deković, M., Cook, W. L., Manders, W., Branje, S. J. T. (2011). Negativity in problematic and nonproblematic families: A multigroup social relations analysis with structured means. Journal of family psychology, 25(1), 152-156. doi:10.1037/a0022450

Eichelsheim, V. I., Deković, M., Buist, K. L., \& Cook, W. L. (2009). The Social Relations Model in Family Studies: A Systematic Review. Journal of Marriage and Family, 71(4), 10521069. doi:10.1111/j.1741-3737.2009.00652.x

Kashy, D. A., \& Kenny, D.A. (1990). Analysis of family research designs: A model of interdependence. $\quad$ Communication Research, 17(4), 462-482. doi:10.1177/009365090017004004

Kenny, D. A., Kashy, D. A., \& Cook, W. L. (2006). Dyadic Data Analysis. New York: The Guilford Press.

Kenny, D. A., \& La Voie, L. (1984). The Social Relations Model. Advances in Experimental Social Psychology, 18, 141-182.

Lüdtke, O., Robitzsch, A., Kenny, D. a., \& Trautwein, U. (2013). A General and Flexible Approach to Estimating the Social Relations Model Using Bayesian Methods. Psychological methods, 18(1), 101-119. doi:10.1037/a0029252

Maccoby, E. E. (2003). Dynamic Viewpoints on Parent Child Relations, their Implications for Socialization Processes. In L. Kuczynski (Ed.), Handbook of dynamics in parent child relations. Thousand Oaks, CA: Sage.

Majdandžić, M., Vente, W. de, Feinberg, M. E., Aktar, E., \& Bögels, S. M. (2012). Bidirectional Associations Between Coparenting Relations and Family Member Anxiety: A Review and 
fSRM: A user-friendly R package for family data | 31

Conceptual Model. Clinical child and family psychology review, 15(1), 28-42. doi:10.1007/s 10567-011-0103-6

Manders, W. A., Cook, W. L., Oud, J. H. L., Scholte, R. H. J., Janssens, J. M. A. M., \& De Bruyn, E. E. J. (2007). Level validity of self-report whole-family measures. Journal of family psychology, 21(4), 605-613. doi:10.1037/0893-3200.21.4.605

Padilla-Walker, L. M., Carlo, G., Christensen, K. J., \& Yorgason, J. B. (2012). Bidirectional Relations between Authoritative Parenting and Adolescents Prosocial Behaviors. Journal of Research on Adolescence, 22(3), 400-408. doi:10.1111/j.1532-7795.2012.00807.x

Rasbash, J., Jenkins, J., O’Connor, T. G., Tackett, J., \& Reiss, D. (2011). A Social Relations Model of Observed Family Negativity and Positivity Using a Genetically Informative Sample. Journal of personality and social psychology, 100(3), 474-491. doi:10.1037/a0020931

Rosseel, Y. (2012). lavaan: An r package for structural equation modeling. Journal of Statistical Software, 48(2).

Schönbrodt, F.D., Back, M.D., \& Schmukle, S.C. (2012). TripleR: An R Package for Social Relations Analyses Based on Round-Robin Designs. Behavior research methods, 44(2), 455-70. ISSN 1554-3528. URL http://www.persoc.net/.

Schönbrodt, F. D., Back, M. D., \& Schmukle, S. C. (2014). TripleR: Social Relation Model (SRM) analyses for single or multiple round-robin groups (version 1.4). Retrieved from http://cran.r-project.org/web/packages/TripleR/index.html

Schönbrodt, F.D., Stas, L., Loeys, T. (2014). fSRM: An R package for Social Relations Models with roles (version 0.6). Retrieved from http://cran.rproject.org/web/packages/fSRM/index.html 


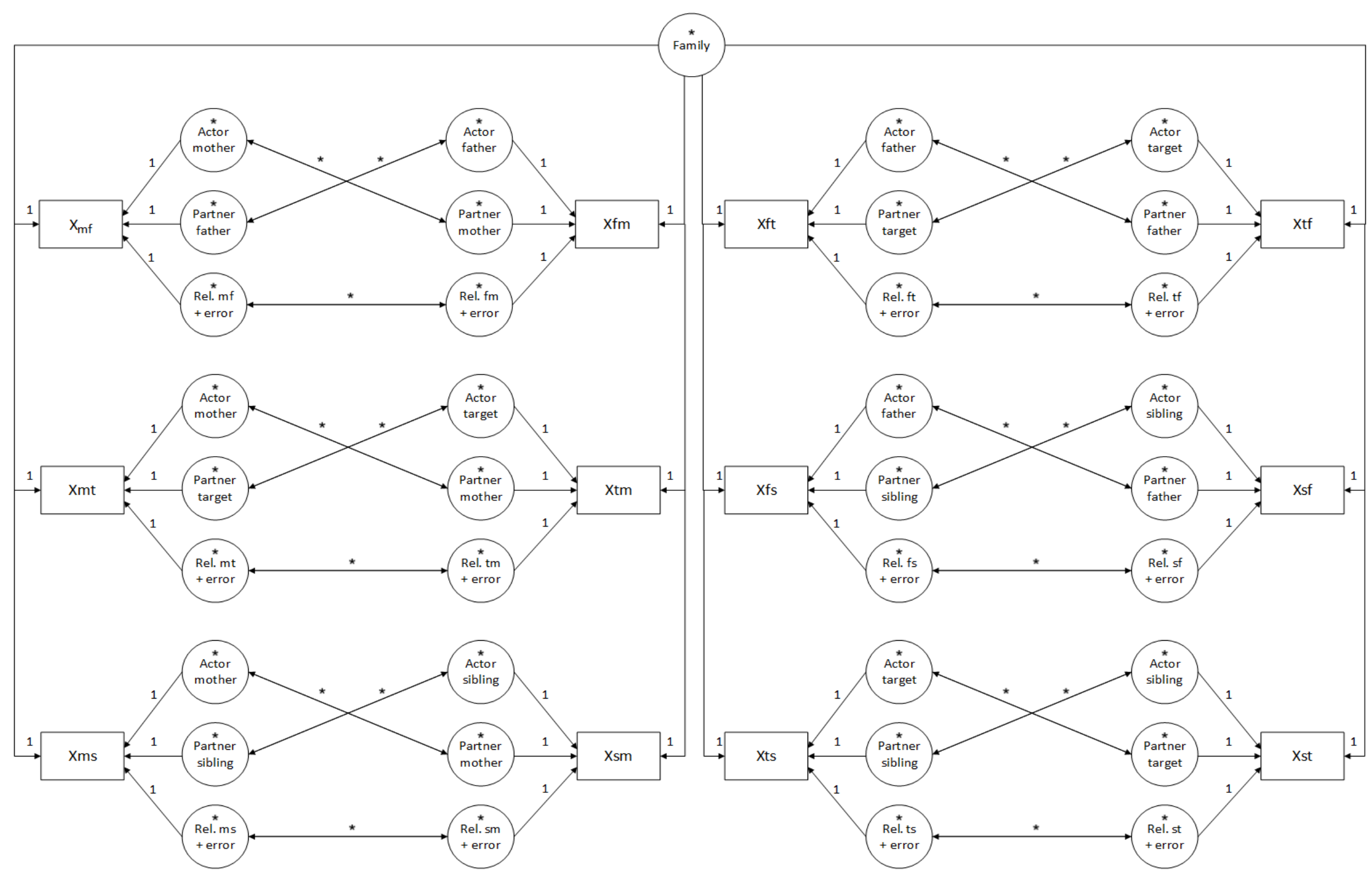

Figure 1: The SRM variances in a CFA. Boxes represent observed dyadic measurements, circles latent variables. Parameters that are fixed are indicated by ' 1 ', free variances by an asterisk. Every indicator is connected with the corresponding latent variable by a single headed arrow. Double headed arrows represent reciprocities. Note that the actor (partner) effect of the same rater (person being rated) are repeated at different places, which is done for clarity. They are assumed to be the same. 
fSRM: A user-friendly R package for family data | 33

\begin{tabular}{|l|l|l|l|l|l|l|}
\hline & family.id & variable & value & actor.id & partner.id & ind \\
\hline 1 & 1 & MFneg & 2.38 & M & F & neg \\
\hline 2 & 2 & MFneg & 1.59 & M & F & neg \\
\hline 3 & 3 & MFneg & 1.55 & M & F & neg \\
\hline
\end{tabular}

\begin{tabular}{|c|l|l|l|l|}
\hline & ID & MFneg & FMneg & MTneg \\
\hline 1 & 1 & 2.38 & 2.84 & 2.66 \\
\hline 2 & 2 & 1.59 & 0.85 & 1.50 \\
\hline 3 & 3 & 1.55 & 1.03 & 2.29 \\
\hline
\end{tabular}

\begin{tabular}{|c|c|c|c|c|c|c|}
\hline 121 & 1 & FMneg & 2.84 & $\mathrm{~F}$ & M & neg \\
\hline 122 & 2 & FMneg & 0.85 & $F$ & M & neg \\
\hline 123 & 3 & FMneg & 1.03 & $F$ & M & neg \\
\hline \multicolumn{7}{|c|}{$\cdots$} \\
\hline 241 & 1 & MTneg & 2.66 & M & $T$ & neg \\
\hline 242 & 2 & MTneg & 1.5 & M & $T$ & neg \\
\hline 243 & 3 & MTneg & 2.29 & M & $T$ & neg \\
\hline
\end{tabular}

Figure 2: Transformation of the clinical dataset from wide to long format. For example, MFneg (left panel) represents the mother rating the father with 2.38 on negativity.

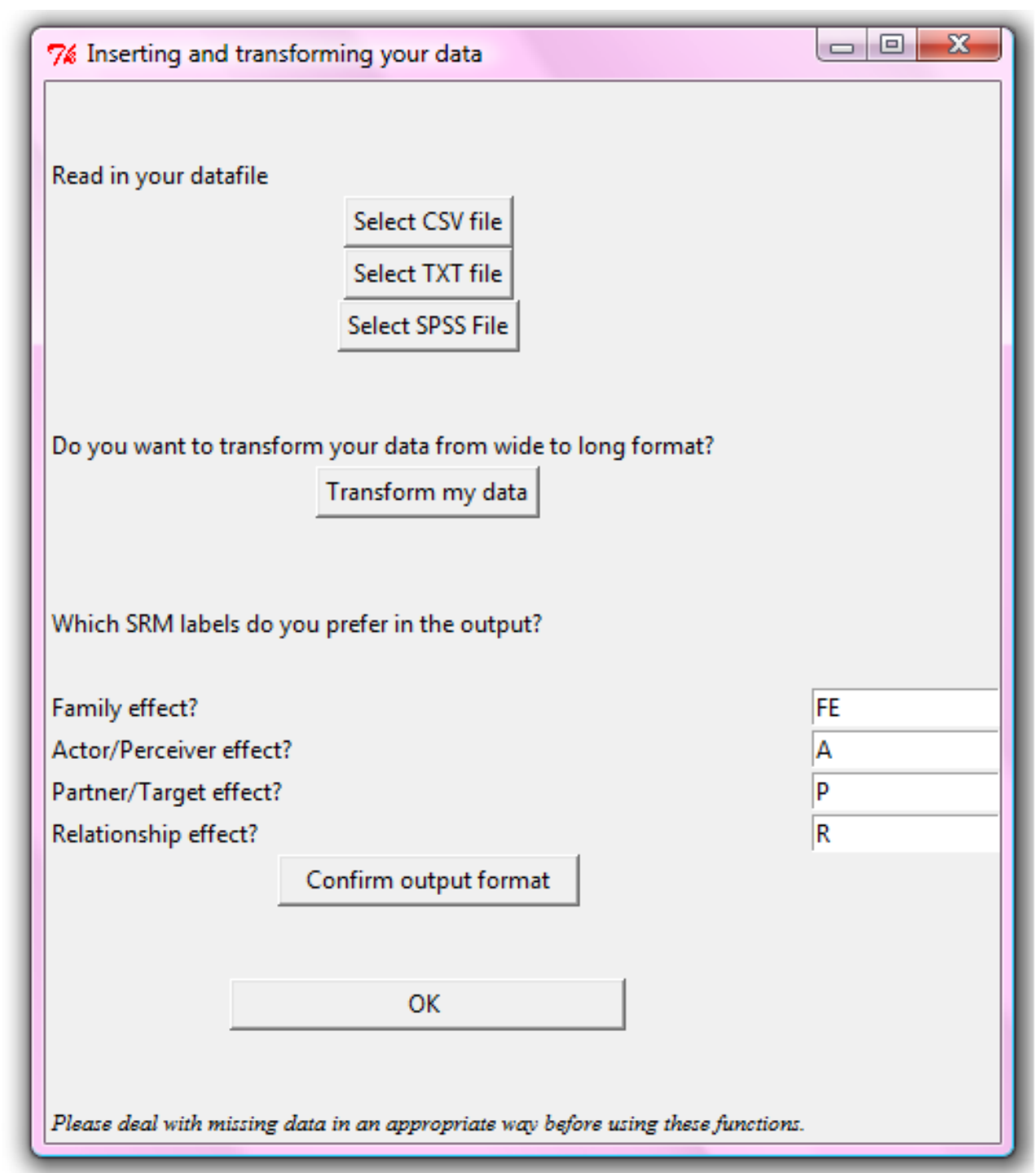

Figure 3: The pop-up window allows to easily read and transform datasets. 
fSRM: A user-friendly R package for family data $\mid 34$

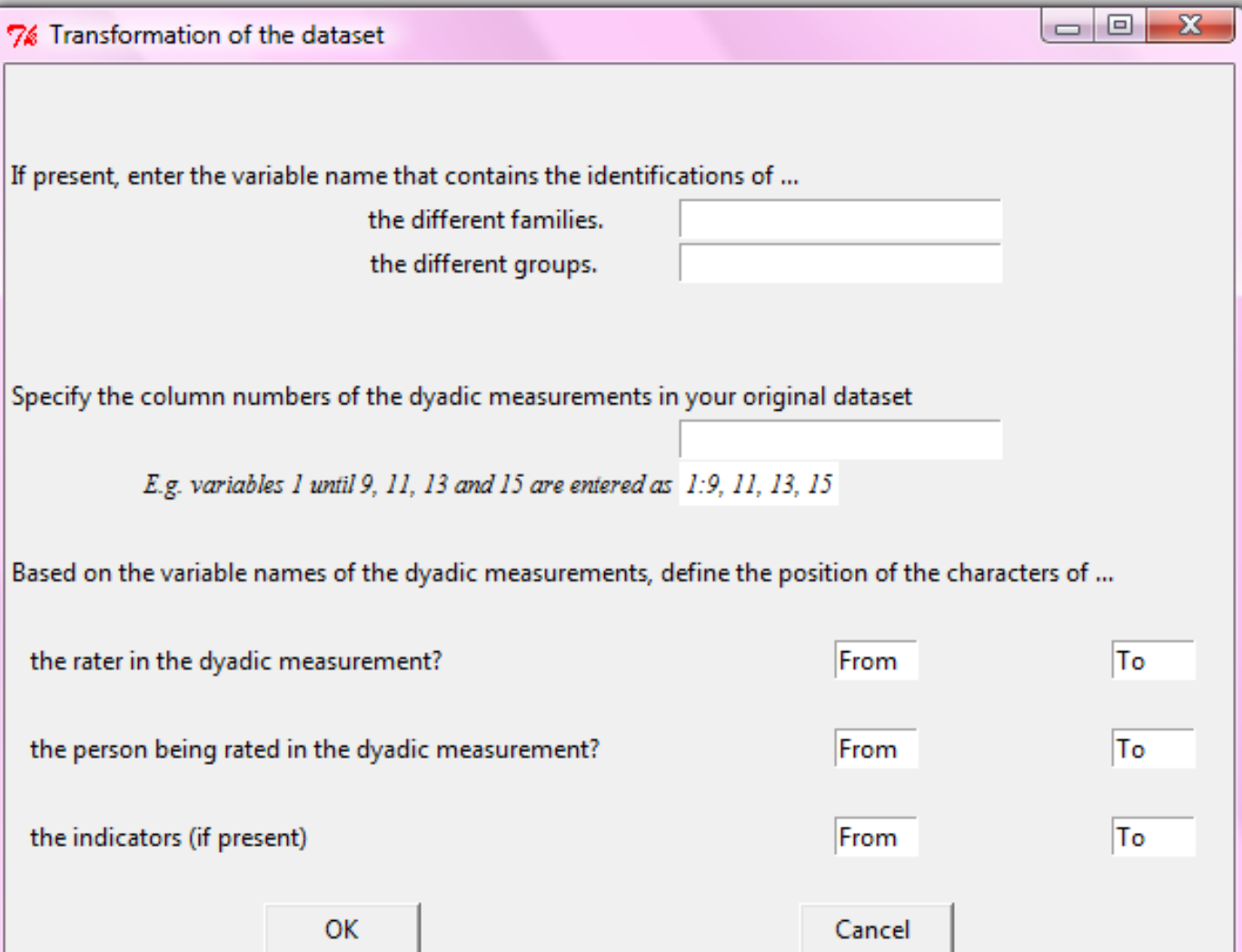

Figure 4: When automatically transforming a dataset from wide to long format, these characters and variables need to be specified by the user.

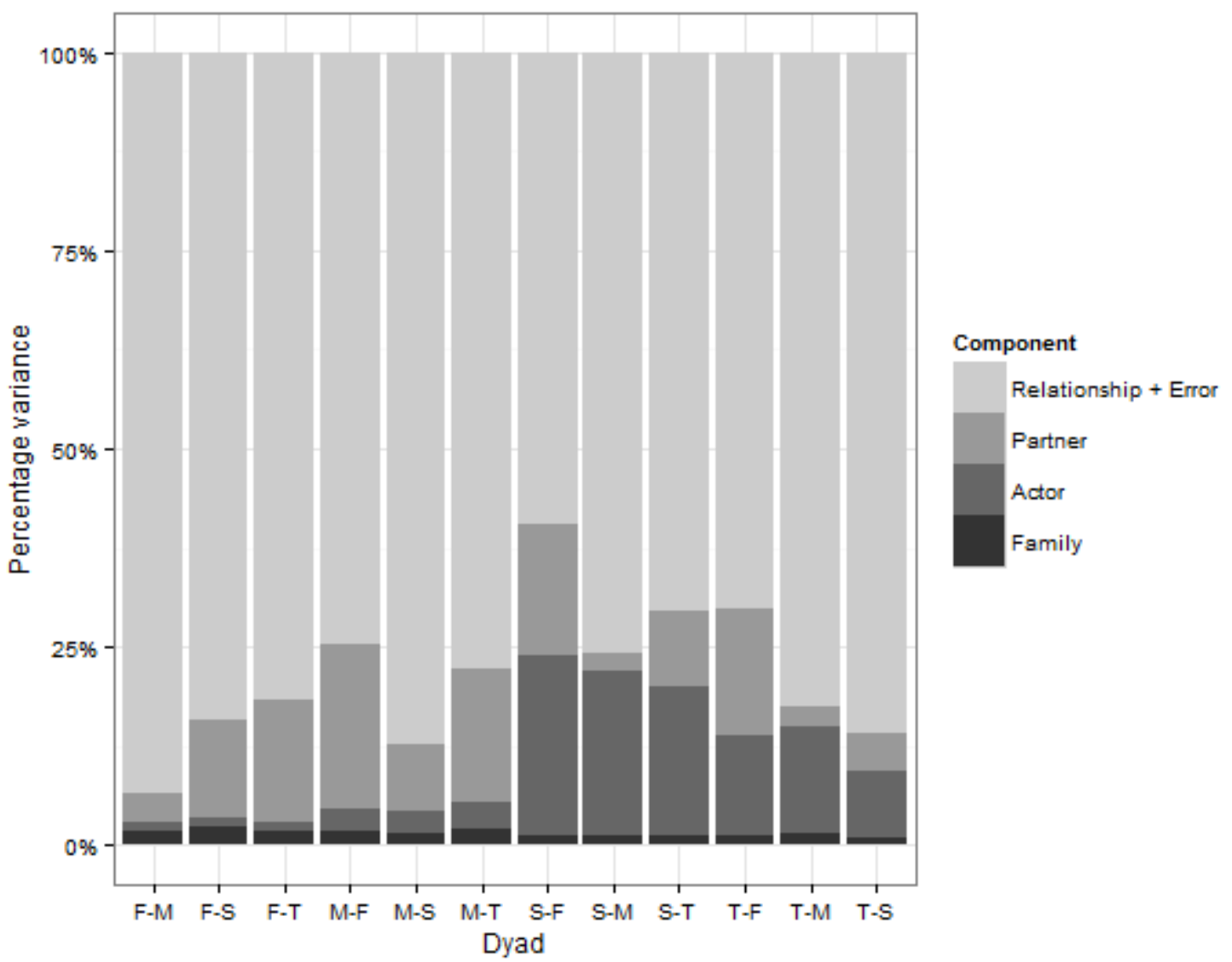


Figure 5: Relative variance decomposition plot. Every bar represents an observed dyadic measurement and shows the relative importance of each SRM variance per observed relationship.

Note. T represents the target adolescent, $\mathrm{M}$ the mother, $\mathrm{F}$ the father and $\mathrm{S}$ the sibling. (E.g., $\mathrm{F}-\mathrm{M}=$ father rating the mother).

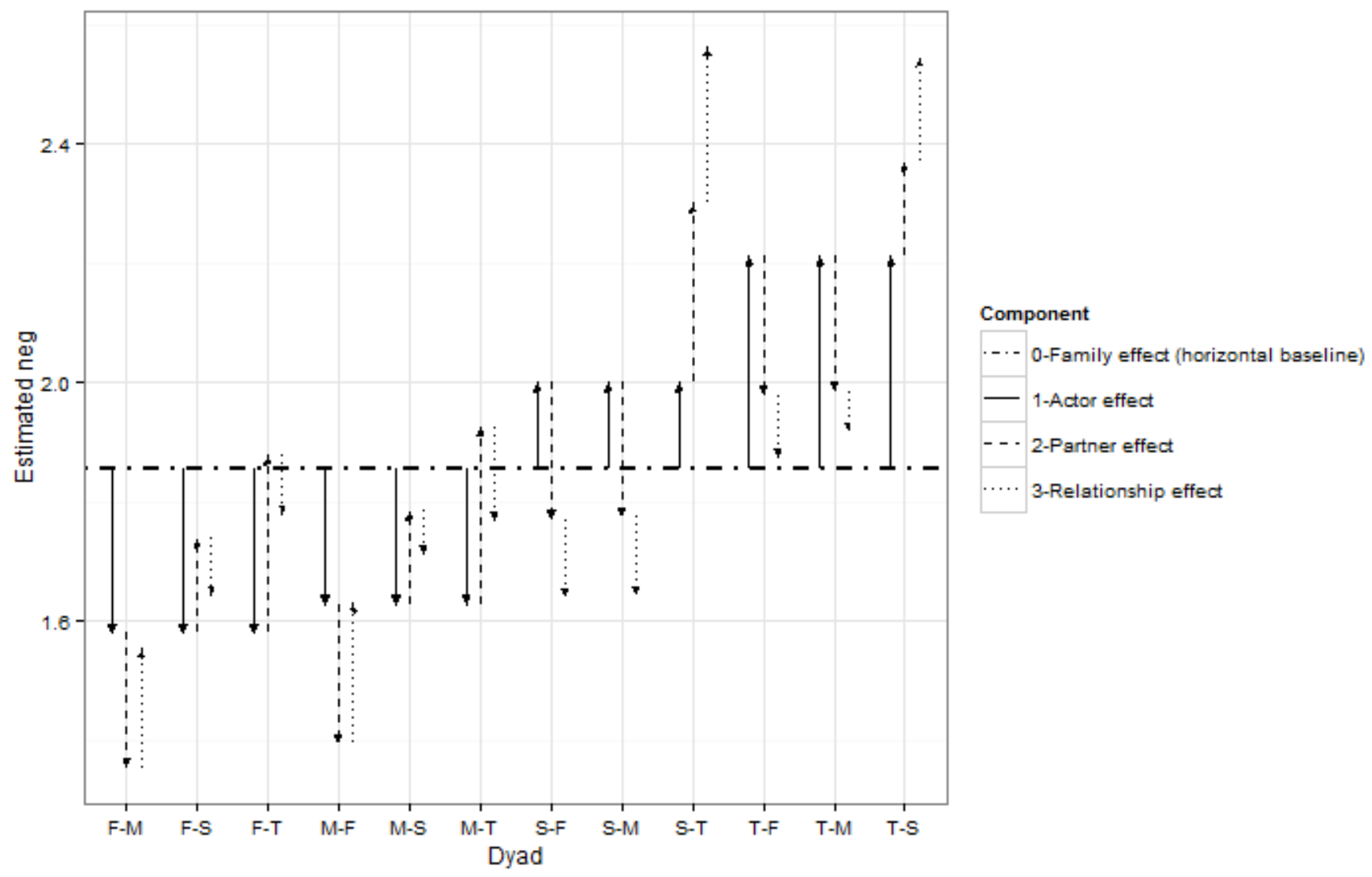

Figure 6: Decomposition of the mean dyadic measurements into the mean SRM components. The character before the hyphen represents the rater (i.e., actor of the dyad), the character after the hyphen the person being rated (i.e., partner of the dyad).

Note. T represents the target adolescent, $\mathrm{M}$ the mother, $\mathrm{F}$ the father and $\mathrm{S}$ the sibling. (E.g., $\mathrm{F}->\mathrm{M}=$ father rating the mother). 